\title{
Wandel der Parteiensysteme in den Ländern Ostmitteleuropas: Stabilität und Effektivität durch Konzentrationseffekte?
}

\author{
Tom Thieme
}

Das Ende der „Vierten Republik“ Polens - beschleunigt durch die Abwahl der Regierung Jarosław Kaczyński in den vorgezogenen Parlamentswahlen am 21. Oktober $2007^{1}$ - wurde von den meisten in- und ausländischen Beobachtern mit Erleichterung registriert. Während sich die europäischen Partner Warschaus in erster Linie eine europafreundlichere Außenpolitik der neuen Koalition unter Führung von Premierminister Donald Tusk und seiner Bürgerplattform (PO) erhoffen, stimmten vor allem viele junge Polen wegen der innen- und wirtschaftspolitischen Stagnation des Landes gegen eine Fortsetzung des konservativ-populistischen Bündnisses unter Führung der Partei Recht und Gerechtigkeit (PiS) der Kaczyński-Zwillinge. ${ }^{2}$ Das Wählervotum für den Wechsel war vor allem eine Abrechnung mit der von Polarisierung und Feindseligkeit gekennzeichneten Politik der Regierung sowie der skandalbehafteten Zusammenarbeit mit den beiden als extremistisch einzustufenden Koalitionspartnern Samoobrona (Selbstverteidigung) und Liga der Polnischen Familien (LPR). ${ }^{3}$

Das Ergebnis der Sejm-Wahlen bestätigt zwei Entwicklungen, deren Ursachen, Auswirkungen und Wechselbeziehungen sich dieser Beitrag widmet: Zum einen überstand bislang keine Regierungskoalition in Polen eine Wahlperiode ohne Kabinettsumbildungen und / oder Neuwahlen; das stützt den Befund „stabiler Instabilität“ in den Parteiensystemen Ostmitteleuropas. Zum anderen unterstreicht das Beispiel Polen die Tendenz einer stetigen Verkleinerung der Parteienanzahl in den ostmitteleuropäischen Parlamenten. Besonders der zweite Aspekt scheint aus politikwissenschaftlich-vergleichender Perspektive interessant zu sein. Daher wird versucht, den Wandel der Parteiensysteme in Polen, Tschechien, der Slowakei und Ungarn seit Beginn der demokratischen Transformation mit Blick auf zunehmende Konzentrationseffekte komparatistisch auszuleuchten. Zentrales Augenmerk liegt auf den Ursachen, die für diese Entwicklung maßgeblich verantwortlich sind und den Auswirkungen, die sich daraus für die Parteiensysteme in den vier Staaten unter Berücksichtigung der Kriterien Regierungsstabilität und Effektivität ergeben. Abschließend wird der Frage nachgegangen, ob und inwieweit die Veränderungen der Strukturmerkmale Format und Fragmentierung - trotz der anhaltenden mehr oder weniger dynamischen Entwicklung in Ostmitteleuropa - in eine Parteiensystemtypologie übertragen werden können. Die Überlegungen orientieren sich am Vorschlag von Alan Ware, der nach der Anzahl der Parteien unter Berücksichtigung ihrer relativen Größe typologisiert. ${ }^{4}$ Eine solche Klassifikation wird dem Untersuchungsgegenstand dieses Beitrags am ehesten gerecht und somit gegenüber Typologien mit ideologisch-inhaltlichen Komponenten (zum Beispiel Giovanni Sar-

1 Vgl. dazu den Beitrag von Holger Münch in diesem Heft der ZParl.

2 Vgl. Peter Oliver Loew, Das Ende der „Vierten Republik“. Die polnischen Parlamentswahlen vom Oktober 2007, in: Osteuropa, 57. Jg. (2007), H. 10, S. 43 - 49.

3 Siehe hierzu Tom Thieme, Hammer, Sichel, Hakenkreuz. Parteipolitischer Extremismus in Osteuropa: Entstehungsbedingungen und Erscheinungsformen, Baden-Baden 2007, S. 107 - 125.

4 Vgl. Alan Ware, Political Parties and Party Systems, Oxford / New York 1996, S. 162. 
tori und Klaus von Beyme) bevorzugt. Die Einbeziehung der ideologischen Polarisierung auf einer Rechts-Links-Achse scheint zudem wenig sinnvoll, da die „eigenartige Ausfransung“5 der klassischen politischen Richtungsbegriffe in Osteuropa deren Anwendung vor kaum überwindbare Abgrenzungsprobleme stellt.

\section{Parlamentarisches Format und Fragmentierung in Ostmitteleuropa seit 1990}

Die vergleichende Untersuchung Polens, Tschechiens, der Slowakei und Ungarns bietet sich zunächst wegen der naturräumlichen Verbindung als Staaten Ostmitteleuropas an. ${ }^{6}$ Allerdings reicht dieser Umstand als relevantes Auswahlkriterium für die vergleichende Analyse der Parteiensysteme nicht aus. Vielmehr weist die politische und marktwirtschaftliche Transformation der vier Staaten wesentliche Parallelen auf, die das Entstehen ähnlich kompetitiver Parteiensysteme begünstigen. Ausgangs- und vorläufiger Endpunkt der postkommunistischen Entwicklung waren in diesen Ländern weitgehend identisch. Bei allen Schwierigkeiten der Umwandlung des gemeinsamen sozialistischen Erbes galten Polen, Tschechien und Ungarn in den 1990er Jahren als Musterländer der Transformation; die Slowakei folgte mit etwas Verspätung. ${ }^{7}$ Sie etablierten früh ähnliche Demokratie- und Marktwirtschaftsmodelle und erreichten das Ziel der EU-Mitgliedschaft am 1. Mai 2004 gleichzeitig - trotz zeitweilig unterschiedlich großer Fortschritte.

Bei der rein quantitativen Bestimmung der ostmitteleuropäischen Parteiensysteme sind - außer in der Slowakei - Veränderungen zugunsten einer zunehmenden Parteienkonzentration festzustellen (vgl. Tabelle 1). Die Gründungswahlen 1990/91 markieren dahingehend Ausnahmen, da ihre Ergebnisse der speziellen Umbruchsituation geschuldet waren. Bei den ersten nichtkommunistisch organisierten Wahlen im gesamten Ostblock in der ČSFR gelang im tschechischen Landesteil nur vier Parteienbündnissen der Einzug in die damalige Volkskammer, was ein klares Votum für das oppositionelle Bürgerforum (OF) und den Systemwechsel unter Führung Václav Havels bedeutete (53,2 Prozent). Das slowakische Pendant Öffentlichkeit gegen Gewalt (VPN) war dagegen nicht so stark geeint wie das OF - hier zogen fünf Parteien in die Parlamentskammer ein. Angesichts von 52 angetretenen Organisationen in beiden Landesteilen bedeutete dies eine deutliche und unerwartete Parteienkonzentration. ${ }^{8}$ In Polen dagegen schien bei den Sejm-Wahlen 1991 der Systemkonflikt nach den halbfreien Wahlen 1989 bereits überwunden. 29 Gruppierungen (elf davon mit je einem Sitz) gelangten ins Parlament, was eine stabile Regierungsbildung bis zu

5 Klaus von Beyme, Rechtsextremismus in Osteuropa, in: Jürgen W. Falter / Hans Gerd Jaschke I Jürgen R. Winkler (Hrsg.), Rechtsextremismus. Ergebnisse und Perspektiven der Forschung, Opladen 1996, S. 423 - 442.

6 Der geographische Begriff „Ostmitteleuropa“ umfasst Polen, Tschechien, die Slowakei und Ungarn sowie den aus der jugoslawischen Föderation hervorgegangenen ehemaligen Teilstaat Slowenien. Dieser wird jedoch auf Grund der speziellen postjugoslawischen Transformationsbedingungen nicht in die Reihe der anderen vier ostmitteleuropäischen Staaten aufgenommen. Vgl. im Einzelnen zum Ost- und Ostmitteleuropabegriff Timothy Garton Ash, History of the Present. Essays, Sketches, and Dispatches from Europe in the 1990s, London u.a. 1999.

7 Vgl. Kai-Olaf Lang, Systemtransformation in Ostmitteleuropa: Eine erste Erfolgsbilanz, in: APuZ, B 15 (2001), S. $13-21$.

8 Vgl. Florian Grotz, Politische Institutionen und postsozialistische Parteiensysteme in Ostmitteleuropa. Polen, Ungarn, Tschechien und die Slowakei im Vergleich, Opladen 2000, S. 318 - 323. 
den Neuwahlen 1993 verhinderte. ${ }^{9}$ Auch in Ungarn war die zentrale inhaltliche Konfliktdimension zum Zeitpunkt der Wahlen 1990 nicht mehr die Einstellung zum Sozialismus, sondern welches postkommunistische Reformkonzept sich durchsetzen sollte. Allerdings brachte das Wahlergebnis 1990 eine wesentlich höhere Konzentration des Parteiensystems als in Polen und als die Kandidaturebene erwarten ließ. Von 54 registrierten Parteien zogen nur sechs ins Budapester Parlament ein, dazu neun direkt gewählte Abgeordnete. ${ }^{10}$

Bis 2007 verkleinerte sich dagegen das parlamentarische Format in allen vier Staaten nahezu konstant (nur in der Slowakei mit geringerem Ausmaß). In Polen schafften 1993, 2001 und 2005 jeweils sechs Parteien beziehungsweise Wahlbündnisse den Sprung über die Fünfprozenthürde und damit den Einzug in den Sejm; 1997 waren es lediglich fünf. Seit Oktober 2007 sind erstmals nur noch vier Parteien im Sejm vertreten, was einen Tiefststand der Anzahl an Fraktionen bedeutet. ${ }^{11}$ Deutlicher noch wird der Befund zur Parteienkonzentration hinsichtlich der Gesamtstimmenanteile der Parteien. Die vier stärksten Parteien nahmen - abgesehen vom Urnengang 1997 - bei jeder Wahl zu. 2007 konnten diese 95,7 Prozent aller Wählerstimmen gewinnen (vgl. Tabelle 2).

In Tschechien verringerte sich die Anzahl der Fraktionen bei den vier Urnengängen von 1992 bis $2002^{12}$ von sechs auf vier. Erst 2006 zog mit der Grünen Partei (SZ) überraschend eine neue und somit fünfte politische Kraft in das tschechische Abgeordnetenhaus ein, wenngleich äußerst knapp (6,3 Prozent). ${ }^{13}$ Dies entspricht dem zweitniedrigsten Stand an Fraktionen in Tschechien seit 1990 (vgl. Tabelle 1). In der ideologisch-programmatischen Strukturierung ähnelt das tschechische Parteiensystem mit zwei dominanten Kräften - Demokratische Bürgerpartei (ODS) und Tschechische Sozialdemokratische Partei (ČSSD) -, der bürgerlich-konservativen Volkspartei (KDU), der nur teilweise reformierten Kommunistischen Partei Böhmens und Mährens (KSČM) sowie den Grünen momentan dem deutschen Fünfparteiensystem. Eine erneute Verringerung kann allerdings bei nur geringfügig schwindender Zustimmung für die Grünen nicht ausgeschlossen werden. Die Stärkeverhältnisse der jeweils vier erfolgreichsten Parteien blieben davon ohnehin unberührt. Die Fragmentierung der Stimmen nahm seit 1992 konstant ab, wenngleich bei den Wahlen 2006 und im Unterschied zu den Verschiebungen in den 1990er Jahren in deutlich geringerem Umfang (vgl. Tabelle 2).

Weniger eindeutig in Richtung zunehmender Parteienkonzentration verlief die Entwicklung in der Slowakei. Bei den vier Parlamentswahlen seit Beginn der slowakischen Unabhängigkeit zogen 1994 und 2002 je sieben Fraktionen in den slowakischen Nationalrat ein; 1998 und 2006 waren es jeweils sechs. Während bei den Wahlen 1998 die vier stärksten politischen Kräfte einen Gesamtanteil von über 77 Prozent der Stimmen gewannen, erreichten sie 2002 zusammen weniger als 60 Prozent. Die Wahl 2006 brachte dahingehend

9 Vgl. Klaus Ziemer / Claudia-Yvette Matthes, Das politische System Polens, in: Wolfgang Ismayr (Hrsg.), Die politischen Systeme Osteuropas im Vergleich, Opladen 2004, S. 189 - 246, S. 217.

10 Vgl. Florian Grotz, a.a.O. (Fn. 8), S. 231 f.

11 Die Partei Deutsche Minderheit (MN) ist zwar mit einem Abgeordneten im Sejm vertreten, wird allerdings wegen der Ausnahmeregelung einer für sie nicht wirksamen Sperrklausel bei der Gesamtbetrachtung der Anzahl der Parteien nicht berücksichtigt.

12 Vgl. dazu auch Andrea Gawrich, Die jüngsten Parlamentswahlen in Polen, Ungarn und Tschechien, in: ZParl, 34. Jg. (2003), H. 2, S. $270-284$.

13 Vgl. Offizielles Statistikamt der Tschechischen Republik, http://www.volby.cz, Abruf am 9. Januar 2008. 
Tabelle 1: Anzahl der Parlamentsfraktionen in Ostmitteleuropa seit 1990/91

\begin{tabular}{|c|c|c|c|c|c|}
\hline Wahljahr & Polen & Tschechien & Slowakei & Ungarn & Median \\
\hline $1990 / 91$ & 29 & $4^{*}$ & $5^{*}$ & 6 & 5,5 \\
\hline 1992 & & $6^{*}$ & $5^{*}$ & & 6,0 \\
\hline 1993 & 6 & & & & 6,0 \\
\hline 1994 & & & 7 & 6 & 6,0 \\
\hline 1996 & & 6 & & & 6,0 \\
\hline 1997 & 5 & & & & 6,0 \\
\hline 1998 & & 5 & 6 & 6 & 5,5 \\
\hline 2001 & 6 & & & & 6,0 \\
\hline 2002 & & 4 & 7 & 3 & 5,0 \\
\hline 2005 & 6 & & & & 5,0 \\
\hline 2006 & & 5 & 6 & 4 & 5,5 \\
\hline 2007 & 4 & & & & 4,5 \\
\hline
\end{tabular}

* Angaben jeweils für den tschechischen und den slowakischen Landesteil bei den Parlamentswahlen in der Tschechoslowakei 1990 und 1992.

Quelle: Eigene Zusammenstellung aus verschiedenen Quellen. Ergebnisse der Parlamentswahlen unter anderem in: Dieter Segert / Ellen Bos (Hrsg.), Osteuropäische Demokratien als Trendsetter? Parteien und Parteiensysteme nach Ende des Übergangsjahrzehnts, Opladen / Farmington Hills 2008.

eine erneute Umkehr der Entwicklung, da sich sowohl die Parteienzahl verringerte (von sieben auf sechs) als auch der Gesamtstimmenanteil der vier Erstplatzierten deutlich zunahm und den zweithöchsten Wert seit Beginn der slowakischen Unabhängigkeit aufweist (vgl. Tabelle 2).

\begin{tabular}{|c|c|c|c|c|c|}
\hline \multicolumn{6}{|c|}{$\begin{array}{l}\text { Tabelle 2: Konzentration der Parteiensysteme in Ostmitteleuropa (Stimmenanteile der vier } \\
\text { stärksten Parteien) }\end{array}$} \\
\hline Wahljahr & Polen & Tschechien & Slowakei & Ungarn & Median \\
\hline $1990 / 91$ & 41,7 & $83,3^{*}$ & $76,3^{*}$ & 68,7 & 72,5 \\
\hline 1992 & & $56,5^{*}$ & $68,8^{*}$ & & 62,6 \\
\hline 1993 & 53,7 & & & & 62,6 \\
\hline 1994 & & & 65,6 & 73,3 & 61,1 \\
\hline 1996 & & 74,4 & & & 69,5 \\
\hline 1997 & 81,6 & & & & 73,9 \\
\hline 1998 & & 80,0 & 77,1 & 83,1 & 80,8 \\
\hline 2001 & 73,4 & & & & 78,6 \\
\hline 2002 & & 87,5 & 59,3 & 93,1 & 80,5 \\
\hline 2005 & 73,8 & & & & 80,7 \\
\hline 2006 & & 87,7 & 71,1 & 99,1 & 80,7 \\
\hline 2007 & 95,7 & & & & 91,7 \\
\hline \multicolumn{6}{|c|}{$\begin{array}{l}\text { * Angaben jeweils für den tschechischen und den slowakischen Landesteil bei den Parlamentswahlen in } \\
\text { der Tschechoslowakei } 1990 \text { und } 1992 . \\
\text { Quelle: Eigene Zusammenstellung aus verschiedenen Quellen (siehe Tabelle 1). }\end{array}$} \\
\hline
\end{tabular}


Ungarn blieb bis 2002 ein verhältnismäßig stabiles Sechsparteiensystem. Gleichwohl nahm die Konzentration der Wählerstimmen bei jeder der fünf postkommunistischen Wahlen seit 1990 zu. Mittlerweile vereinen die vier stärksten Parteien 99,1 Prozent der abgegebenen Wählerstimmen - ein Rekordwert in Ost- und Westeuropa. Dies wirkte sich allerdings erst seit 2002 auch auf eine Abnahme der im Parlament vertretenen Kräfte aus. Bei den letzten beiden Wahlen gelang drei Parteien beziehungsweise Parteiallianzen ${ }^{14}$ der Sprung über die Sperrhürde von fünf Prozent für Einzelparteien und zehn Prozent für Bündnisse. Nur dank einer landesweiten Ausgleichsliste, die im komplizierten ungarischen Wahlsystem zur Kompensation mehrheitsbildender und damit große Parteien begünstigender Effekte angewendet wird, zogen auch elf Abgeordnete des Ungarischen Demokratischen Forums (MDF) ins Parlament ein, so dass sich die Zahl der Einzelfraktionen von drei auf vier erhöhte. ${ }^{15}$ Aktuelle Umfragen in Ungarn sehen hingegen beide kleinen Parteien unterhalb der Sperrklausel, was die erwartete Entwicklung in Richtung eines Zweiparteiensystems und damit einer weiteren Parteienkonzentration bestätigt. ${ }^{16}$

Bei der Gesamtbetrachtung der vier ostmitteleuropäischen Staaten treten die Befunde zur Verkleinerung des parlamentarischen Formats und zur Parteienkonzentration noch deutlicher zu Tage. Ohne die Sonderfälle der jeweils ersten freien Wahl zu berücksichtigen, ist die Entwicklung in allen vier Staaten offensichtlich: Abgesehen von geringen Abweichungen - deren Erklärung im folgenden Kapitel gleichwohl nicht vernachlässigt werden darf - vereinen immer weniger Parlamentsparteien immer größere Anteile von Wählerstimmen. Die Konzentration der Parteiensysteme der vier Länder nahm seit 1993 konstant zu - lediglich die polnischen Sejm-Wahlen 2001 und die Nationalratswahlen 2002 in der Slowakei markieren dahingehend Ausnahmen. Eine Abnahme der Durchschnittswerte vollzog sich allerdings nur nach der Wahl 2001 in Polen, da 2002 die abnehmende Parteienkonzentration in der Slowakei mit einer starken Verschiebung der Kräfteverhältnisse zugunsten wenigerer Parteien in Ungarn und Tschechien einherging. Einfacher noch ist die Entwicklung mit bloßem Blick auf das Parteiensystemformat zu erkennen: Die Anzahl an Fraktionen sank laut Medianwerten von 1994 (6,5), 1998 (5,5), 2002 (5,0) fast konstant auf mittlerweile weniger als fünf Parteien (2007: 4,5; vgl. Tabelle 1). Damit liegt die Anzahl an parlamentarisch vertretenen Parteien in Ostmitteleuropa unter dem analogen aktuellen Wert für Westeuropa. Dieses Quantum ist besonders auffallend, denn die parlamentarische Fragmentierung ist in der Mehrzahl der westeuropäischen Staaten in den vergangenen zwanzig Jahren fortlaufend gestiegen, während sie in Ostmitteleuropa gegen den Trend und kontinuierlich abnahm. ${ }^{17}$

14 Bei den Parlamentswahlen 2002 trat das Ungarische Demokratische Forum (MDF) zusammen mit dem Bund der Jungen Demokraten (FIDESZ) als Zentrumspartei an.

15 Vgl. Edwin Csizmadia, Ungarn sucht den Superstar. Die ungarischen Parlamentswahlen 2006, in: Osteuropa, 56. Jg. (2006), H. 5, S. $75-86$.

16 Ein weiteres Direktmandat gewann ein Vertreter der kroatenungarischen Minderheit, dessen Vereinigung Somogyert bei der Zusammenstellung der Parlamentsparteien in Ungarn unberücksichtigt bleibt. Vgl. Nationales Wahlbüro Ungarns, http://valasztas.hu, Abruf am 10. Januar 2008.

17 Vgl. im Einzelnen Oskar Niedermayer / Richard Stöss / Melanie Haas, Parteiensysteme in Westeuropa: Stabilität und Wandel, in: dies. (Hrsg.), Die Parteiensysteme Westeuropas, Wiesbaden 2006, S. $7-37$, S. $24 \mathrm{f}$. 


\section{Ursachen der Parteienkonzentration}

Um die Gründe einer zunehmenden Parteienkonzentration in Ostmitteleuropa zu erklären, scheint es zweckmäßig, drei Faktorengruppen zu unterscheiden. Zunächst sind die Auswirkungen spezieller politisch-institutioneller Rahmenbedingungen zu nennen. Ein Überblick der politikwissenschaftlich-vergleichenden Literatur zeigt eine deutliche Tendenz für Erklärungsansätze des Parteiensystemwandels in Osteuropa im Polity-Bereich. ${ }^{18}$ Da die Fragmentierung jedoch in den letzten zehn Jahren weiter nachließ, ohne dass nennenswerte Reformen der institutionellen Rahmenbedingungen in den vier Staaten durchgesetzt wurden, trägt dieser eindimensionale Blickwinkel nur bedingt zur Begründung des Parteiensystemwandels in Ostmitteleuropa bei. Florian Grotz differenziert plausibel zwei weitere Erklärungsansätze: gesellschaftsstrukturelle Einflussgrößen, speziell die jeweils historischpolitischen Konfliktlinien, und handlungsbezogene Ursachen wie Organisations- und Verhaltensformen der politischen Akteure. ${ }^{19}$

\subsection{Politisch-institutionelle Rahmenbedingungen}

Politisch-institutionelle Erklärungsansätze für eine abnehmende Fragmentierung sind in erster Linie die Wahlsysteme im Allgemeinen, die Wahlkreisgröße sowie die Sperrklausel im Speziellen. Die mehrheitsbildenden und somit konzentrationsfördernden Effekte von Mehrheits- gegenüber Verhältniswahlsystemen sind theoretisch anerkannt und wurden empirisch mannigfaltig nachgewiesen. ${ }^{20}$ Die Analyse der osteuropäischen Parteiensysteme ergibt jedoch keine klare Übereinstimmung mit den klassischen Erwartungen. Dieter Nohlen kommt zu dem Befund, „unter den gegebenen embryonalen Parteienverhältnissen ist die Mehrheitswahl mit mehr Fragmentierung der Parteienlandschaft verbunden als die Verhältniswahl“21. Für die Parteiensysteme Ostmitteleuropas besitzt die Unterscheidung der Wahlsysteme zudem geringe Bedeutung, da - abgesehen von den jeweils nationalen Sperrhürden - nur das kompensatorische Wahlsystem Ungarns klassische Elemente der Mehrheitswahl enthält. Hier werden jedoch die Effekte der Mehrheitswahl durch Verhältniswahlelemente ausgeglichen. Die direkte Wirkung des Wahlsystems auf die Konzentration des ungarischen Parteiensystems kann nicht zuletzt deswegen bezweifelt werden, weil sich die Verringerung der Anzahl an Parteien erst mit deutlicher Verzögerung gegenüber der Einführung des jeweiligen Wahlmodus einstellte. Zu einem ähnlichen Ergebnis kommt Karsten Schmitz. Seine Untersuchung der Wahlsysteme Osteuropas zeigt, dass der entscheidende Konzentrationseffekt von einem Verhältniswahlrecht mit landesweiter Sperrklausel ausgeht. ${ }^{22}$ Dies lässt sich für Polen und Ungarn nachweisen. Die Zersplitterung im Parla-

18 Vgl. unter anderem Timm Beichelt, Demokratische Konsolidierung im postsozialistischen Europa. Die Rolle der politischen Institutionen, Opladen 2001; Guido Tiemann, Wahlsysteme, Parteiensysteme und politische Repräsentation in Osteuropa, Wiesbaden 2006.

19 Vgl. Florian Grotz, a.a.O. (Fn. 8), S. 445.

20 Vgl. hierzu Arend Lijphart, Patterns of Democracy. Government Forms and Performance in Thirty-Six Countries, New Haven 1999.

21 Dieter Nohlen, Wahlrecht und Parteiensystem, 3. überarbeitete Auflage, Opladen 2000, S. 227.

22 Vgl. Karsten Schmitz, Wahl- und Parteiensysteme in Osteuropa: Eine Neubewertung anhand des Konzentrationseffekts, in: ZParl, 37. Jg. (2006), H. 2, S. 353 - 376, S. 374. 
ment nach den Sejm-Wahlen 1991 verhinderte eine stabile Regierungsbildung, woraufhin eine nationale Sperrklausel von fünf Prozent eingeführt wurde. ${ }^{23}$ Die Anzahl der Fraktionen sank von 29 auf sechs (beziehungsweise sieben bei Einbeziehung der Partei Deutsche Minderheit); ein erneutes Ansteigen der Fragmentierung blieb aus.

In Ungarn wurde die landesweite Hürde für den Einzug ins Parlament 1994 von vier auf fünf Prozent erhöht. Zudem gilt seitdem für Bündnisse aus zwei Parteien eine Sperrklausel von zehn Prozent, bei mehr als zwei Parteien sind es 15 Prozent. ${ }^{24}$ Die Neuregelungen brachten den erhofften Effekt, wenngleich mit zeitlicher Verzögerung. 2002 scheiterte die rechtsextremistische Ungarische Wahrheits- und Lebenspartei (MIÉP) mit 4,4 Prozent an der Fünf-Prozent-Klausel - nach dem alten Wahlgesetz wäre sie im Parlament vertreten gewesen. Kleinere Parteien wie die MDF und die Ungarische Marktpartei (MPP) versuchten ihr Glück in Listenverbindungen mit dem FIDESZ. Oder sie wurden wie die rechtspopulistische Unabhängige Partei der Kleinwirte (FKGP) von ihrem einstigen Seniorpartner vollständig „geschluckt“.

Ähnliche Wahlrechtsänderungen erhöhter und gestaffelter Sperrklauseln in der Tschechoslowakei führten bei den letzten gemeinsamen Wahlen beider Volksgruppen jedoch nicht zu dem erhofften Effekt. Im Gegenteil: Sowohl die Parteienanzahl in Tschechien als auch die Fragmentierung in beiden Landesteilen erhöhte sich. Erst die lange umstrittene Wahlrechtsreform in Tschechien brachte die gewünschte Zunahme der Parteienkonzentration nach der Parlamentswahl 2002. Für Wahlbündnisse aus zwei und mehr Parteien wurde die gestaffelte Sperrklausel von fünf, sieben, neun und elf Prozent zugunsten einer gespreizten Fünfprozenthürde ersetzt, die seitdem für jede Einzelpartei innerhalb eines Bündnisses gilt. Für die zuvor neu gegründete Wahlkoalition aus Freiheitsunion (US), Bürgerpartei (KDU) und zwei kleineren Partnern bedeutete dies zahlreiche „verlorene Stimmen“, da nur die Voten für die beiden großen Partner in Mandatsanteile transferiert wurden. ${ }^{25}$

Das slowakische Wahlrecht erfuhr 1998 tiefgreifende Veränderungen. Diese gehen auf die Absichten des damaligen autoritären Ministerpräsidenten Vladimir Mečiar zurück, seine Macht im Fünf-Millionen-Einwohner-Staat dauerhaft zu sichern. Die bis dahin gültigen vier Wahlbezirke wurden zu nur einem landesweiten Wahlkreis zusammengefasst, um Parteien mit regionalen Hochburgen zu schwächen. Bestanden bis dahin gestaffelte Sperrklauseln für Einzelparteien und Wahlkoalitionen (fünf Prozent für eine Partei, sieben Prozent für Zweier- und Dreierbündnisse, zehn Prozent bei Allianzen mit vier oder mehr Partnern), mussten bei den Parlamentswahlen nun alle Parteien eines Bündnisses mindestens fünf Prozent der landesweiten Wählerstimmen gewinnen. Die Rechnung ging bekanntlich nicht auf: Mečiars Bewegung für eine demokratische Slowakei (HZDS) unterlag der demokratischen Regenbogenkoalition unter Führung Mikuláš Dzurindas. Seine Regierungskoalition bestand aus vier Parteien, der wiederum zahlreiche Vereinigungen vorausgegangenen waren.

23 Vgl. Dieter Bingen, Polen: Wie ein labiles Parteiensystem zu einer Stabilisierung der Demokratie beiträgt, in: Dieter Segert / Ellen Bos (Hrsg.), Osteuropäische Demokratien als Trendsetter? Parteien und Parteiensysteme nach Ende des Übergangsjahrzehnts, Opladen / Farmington Hills 2008, S. 77 - 90, S. 78.

24 Vgl. Csilla Machos, Das postsozialistische Parlament in Ungarn - Strukturen und Akteure, in: Susanne Kraatz / Silvia von Steinsdorff(Hrsg.), Parlamente und Systemtransformation im postsozialistischen Europa, Opladen 2002, S. 63 - 86, S. 82.

25 Vgl. Philipp Harfst, Wahlsystemwandel in Mittelosteuropa. Strategisches Design einer politischen Institution, Wiesbaden 2007, S. 162. 
Insgesamt gelang sechs Parteien der Einzug ins Parlament. Die Wahlreformen verfehlten damit ihre Wirkung.

Dieses Ergebnis offenbart, dass rein politisch-institutionelle Faktoren im Einzelfall für verallgemeinerbare Aussagen über die Ursachen der Parteienkonzentration nicht ausreichen und monokausale Erklärungen zu Fehlschlüssen führen können. Die erzwungenen Allianzen zwischen den demokratischen Kräften in der Slowakei forcierten nicht deren Selbstauflösung, sondern schweißten diese in ihrer gemeinsamen Ablehnung gegen die als undemokratisch angesehene Führungspraxis Mečiars und seiner Getreuen erst zusammen. Die Machtkonstellationen in den einzelnen Ländern sind aufgrund der speziellen historischnationalen Kontexte und der gesellschaftlichen Konfliktlinien nicht gänzlich durch spezifische Institutionenarrangements zu erklären.

\subsection{Gesellschaftsstrukturelle Determinanten}

Im polnischen Parteiensystem der 1990er Jahre stand das postkommunistische Reformbündnis Allianz der demokratischen Linken (SDL) zahlreichen Gruppierungen des nationalen Spektrums gegenüber, die sich im Wahlbündnis Solidarność (AWS) organisiert hatten. Nach der Zersplitterung des AWS und dem Sieg der abgespaltenen PiS bei den Parlamentswahlen 2005 konstruierten die Köpfe der Kaczyński-Partei eine neue Konfliktlinie, die auf den kurzen Nenner „wir und die anderen“ gebracht werden kann. Die feindselige und extrem polarisierende Politik der PiS mobilisierte und konzentrierte die Stimmen auf beiden Seiten des politischen Spektrums. Hatten die vier stärksten Parteien in Polen 2005 zusammen 73,8 Prozent der Stimmen erzielt, waren es 2007 über 95 Prozent, und auch nur diese schafften die Wiederwahl in den Sejm.

In Ungarn ist die gesellschaftliche Polarisierung noch gravierender. Das Cleavage postkommunistisch vs. antikommunistisch spaltet Ungarn in zwei annähernd gleich starke politische Lager. Die bei der Wahl 2006 bestätigte sozialliberale Koalition aus der reformierten Ungarischen Sozialistischen Partei (MSZP) und der SZDSZ vereint nahezu alle linken Kräfte und lässt wenig Raum zur Etablierung am äußeren Rand. Der rechtskonservative FIDESZ bindet - abgesehen von dem ebenfalls konservativen MDF - fast das gesamte bürgerlich-nationale Lager. Sowohl kleinere Parteien der Mitte als auch am rechten und linken Ende des politischen Spektrums haben daher wenig Mobilisierungschancen. Beide Volksparteien laufen so Gefahr, selbst Heimat politisch fragwürdiger Kräfte zu werden.

In Tschechien scheinen gegenläufige Konfliktlinien nicht dieselbe Bedeutung zu haben wie in den Nachbarstaaten. Der Regimekonflikt gilt größtenteils als überwunden und die politisch isolierte KSČM spielt trotz ihrer stabilen Anhängerschaft von zehn bis 15 Prozent in den entscheidenden gesellschaftlichen Debatten nur eine Nebenrolle. Da weder nennenswerte Minderheiten in Tschechien leben, noch konfessionelle Unterschiede im größtenteils entchristianisierten Land existieren, stellt die wirtschaftspolitische Positionierung der Parteien das wichtigste Cleavage dar. ${ }^{26}$ In der Slowakei sind dagegen deutlich heterogenere gesellschaftliche Kräfteverhältnisse vorzufinden. Bereits kurze Zeit nach Beginn des

26 Vgl. Zdenka Mansfeldová, Wahlen im Transformationsprozeß der Tschechischen Republik, in: Klaus Ziemer (Hrsg.), Wahlen in postsozialistischen Staaten, Opladen 2003, S. 283 - 305, S. $292 \mathrm{f}$. 
Systemwechsels beschleunigte sich die Differenzierung verschiedener sozialer Gruppen. Neben die Auseinandersetzungen zwischen alten und neuen Eliten traten Konflikte um die slowakische (Un-)Abhängigkeit, um Konfessionszugehörigkeit und den Status der starken ungarischen Minderheit. Zwar überlagert seit den 1990er Jahren das Cleavage demokratisch vs. autoritär die anderen gesellschaftlichen Konfliktlinien, doch die einzelnen Interessengruppen werden im Parlament nach wie vor von verschiedenen Parteien vertreten.

\subsection{Akteursverhalten}

Der dritte, wenngleich konzeptionell schwieriger zu greifende Erklärungsansatz für die zunehmende Parteienkonzentration in Ostmitteleuropa liegt im Bereich der individuellen Akteursebenen. Verhaltensweisen und Organisationsformen der politischen Eliten trugen in allen vier Ländern ganz unterschiedlich zur Strukturierung des Parteiensystems bei. Die harte Polarisierung zwischen den postkommunistischen und antikommunistischen Lagern in Polen und Ungarn wirkte in drei von vier Fällen defragmentierend. In Ungarn hatten beide Großparteien MSZP und der FIDESZ eine Art Sogwirkung auf kleinere Parteien des jeweiligen Lagers. Abgesehen von der Abspaltung orthodoxer Kommunisten zur bedeutungslosen Arbeiterpartei (MP) einigten Ungarns Reformkommunisten dank ihrer noch immer gut funktionierenden Organisationsstrukturen nahezu alle Anhänger und Vertreter der so genannten einstigen sozialistischen Mittelschicht. Dem FIDESZ schlossen sich nach und nach bürgerliche, christlich-konservative und nationalistische Politiker anderer Fraktionen an. ${ }^{27}$ Diesen Aderlass überlebte keine der kleineren Parteien. Entweder wurde deren Konkursmasse ebenfalls vom FIDESZ geschluckt, oder sie verschwanden gänzlich von der politischen Bühne. Die zur Sozialdemokratie gewendeten ehemaligen Sozialisten Polens wandelten im Jahr $2000 \mathrm{ihr}$ formales Bündnis der Demokratischen Linken (SLD) in eine Partei um und forcierten die Parteienkonzentration im linken Lager. Dagegen nahm zur selben Zeit auf der rechten Seite des Parteienspektrums die Integrationswirkung der Solidarność-Bewegung ab. Die programmatischen, aber auch persönlichen Differenzen der verschiedenen Strömungen und Spitzenkräfte führten zur Zersplitterung des Wahlbündnisses. Die daraus hervorgegangenen Neugründungen PiS und PO dominieren heute den polnischen Parteienwettbewerb.

Für den Niedergang kleinerer Parteien und die damit verbundene Parteienkonzentration sind deren Spitzenpolitiker teilweise auch selbst verantwortlich. Diese Selbstzerstörung trifft vor allem auf die (rechts-)extremen und populistischen Parteien Ostmitteleuropas zu. In den 1990er Jahren feierten sie ihre größten Erfolge und schürten in Westeuropa bisweilen hysterische Befürchtungen vor einem ausufernden radikalen Nationalismus. So rasant die Parteien aufstiegen, so steil ging es für die meisten Extremisten nach kurzer Zeit bergab. Die tschechischen Republikaner stürzten 1998 in eine Finanzkrise, nachdem die Parteispitze immer wieder mit verschwenderischen Investitionen in Privathäuser und Luxusautos für Negativschlagzeilen gesorgt hatte. Die Partei geriet in einen Steuerhinterziehungsskandal

27 Vgl. András Körösenyi / Gábor G. Fodor, Das politische System Ungarns, in: Wolfgang Ismayr (Hrsg.), a.a.O. (Fn. 9), S. $323-372$, S. 350 f. 
und musste im Jahr 2000 Konkurs anmelden. ${ }^{28}$ Zudem wollten nach dem schlechten Abschneiden bei den Wahlen 1998 nicht alle Führungspersonen der Republikaner den uneingeschränkten Machtanspruch und aggressiven Kurs ihres als autoritär und herrisch geltenden Vorsitzenden Miroslav Sládek künftig akzeptieren. Es kam zum Zerwürfnis und zur Zersplitterung der Partei. Aufgrund der fehlenden Finanzmittel für eine Erfolg versprechende Wahlkampagne verzichteten die Überbleibsel der Republikaner darauf, bei der Wahl zum Abgeordnetenhaus 2006 anzutreten. In der ungarischen Kleinlandwirte-Partei (FKGP) kam es Ende der 1990er Jahre zu mehreren Skandalen, weil Teile der Funktionäre den Führungsstil ihres autoritären Vorsitzenden József Torgyán nicht länger hinnehmen wollten. Sie erzielte bei den Wahlen 2002 weniger als ein Prozent der Stimmen; die Reste der Partei wurden wenig später in den FIDESZ integriert. Bei den Wahlen 2007 in Polen stürzten beide extremistischen Parteien ab und verschwanden mit Ergebnissen unter zwei Prozent von der Bildfläche. Samoobronas Vorsitzender Andrzej Lepper stolperte über - offensichtlich inszenierte - Bestechungs- und Sexaffären. Die LPR scheiterte an den Eskapaden und peinlichen Auftritten ihres Spitzenpersonals. Die Slowakische Nationalpartei (SNS) überstand als einzige rechtsextreme Vereinigung in Ostmitteleuropa eine tiefe Krise, ohne längerfristig davon Schaden zu nehmen. Aufgrund interner Machtkämpfe und persönlicher Rangeleien zwischen dem von 1994 bis 1999 amtierenden Vorsitzenden Ján Slota und seiner Nachfolgerin Anna Maliková spaltete sich die Partei aber. Als Konsequenz aus dem Zerwürfnis scheiterten beide Abspaltungen 2002 an der Fünfprozenthürde. Wieder vereint erzielte sie 2006 dank der Reformmüdigkeit vieler slowakischer Wähler das Rekordergebnis von 11,7 Prozent. Die SNS und die tschechische KSČM sind damit die letzten antidemokratischen Parteien in den ostmitteleuropäischen Parlamenten.

\subsection{Unterschiedliche Wirkungen der Ursachenbündel}

Alle drei Ursachenbündel prägten also bislang den Parteienwettbewerb Ostmitteleuropas in Richtung zunehmender Konzentration, gleichwohl in unterschiedlicher Qualität. Die Veränderung der institutionellen Rahmenbedingungen und der gesellschaftlichen Konfliktstrukturen beeinflussten vor allem in der demokratischen Konsolidierungsphase den Parteienwettbewerb. Die Slowakei stellt dahingehend den Sonderfall dar, dass systemimmanente Cleavages erst mit Verzögerung und nach Überwindung des Konflikts demokratisch vs. autoritär entstanden. Seit dem Ende der 1990er Jahre sind vor allem handlungsbezogene Ursachen (Parteiauflösungen, Umformierungen, Neugründungen) für die Konzentration des Parteienwettbewerbs verantwortlich. Dies trifft vor allem auf Polen und die Slowakei zu, deren wichtigste Parteien erst in den vergangenen zehn Jahren entstanden.

\section{Auswirkungen der Parteienkonzentration}

Die Instabilität der ostmitteleuropäischen Regierungskoalitionen wurde vor allem in Untersuchungen zu den 1990er Jahren vielfach auf die relativ hohe Parteienzersplitterung zurückgeführt. Sie folgt der These Giovanni Sartoris, wonach eine größere Anzahl effektiver

28 Vgl. Miroslav Mareš, Pravicový extremismus a radikalismus v ČR, Brno 2003, S. 73 f. 
Parteien, verbunden mit einer erhöhten parlamentarischen Fragmentierung, die Entstehung eines multipolaren Parteiensystems mit verschiedenen Konfliktdimensionen begünstigt. ${ }^{29}$ Zum einen steigt damit die Notwendigkeit, Mehrparteienkoalitionen bilden zu müssen. Umso schwieriger gestaltet sich die Kompromissfindung der Regierungspartner in Sachfragen, was die Wahrscheinlichkeit der vorzeitigen Regierungsauflösung begünstigt. ${ }^{30}$ Zum anderen eröffnen sich den einzelnen Akteuren mehrere Koalitionsoptionen. Dies führt im Fall einer Regierungskrise zur schnelleren Beendigung der Zusammenarbeit als in Parteiensystemen, in denen keine weiteren potenziellen Bündnispartner zur Verfügung stehen.

Für die Regierungskoalitionen in den Staaten Ostmitteleuropas müsste folglich aus der zunehmenden Verkleinerung der Parteiensystemformate eine wachsende Kabinettsstabilität resultieren. Ein Überblick der bisherigen Koalitionen bestätigt diese Annahme. In den hochgradig konzentrierten Parteiensystemen Ungarns und Tschechiens blieben - mit Ausnahme der Mitte-Rechts-Koalition unter Vacláv Klaus (1994 bis 1996) und der im April 2008 gescheiterten Mitte-Links-Regierung unter Ferenc Gyurcsany - alle Regierungsbündnisse bis zum Ende der regulären Wahlperioden bestehen. In den stärker fragmentierten Parteiensystemen in Polen und der Slowakei überstanden dagegen die meisten Koalitionen ihre offiziellen Amtszeiten nicht ohne Kabinettsumbildungen, vorgezogene Neuwahlen oder zumindest ernsthafte Krisen. Der Zusammenhang zwischen der Parteienanzahl und der Lebensdauer ostmitteleuropäischer Regierungskoalitionen unterstreicht dieses Ergebnis: je mehr Fraktionen, desto größer die Wahrscheinlichkeit einer irreparablen Koalitionskrise. Oder anders formuliert: Mit sinkender Parteienanzahl steigt das Stabilitätsniveau der Regierungskoalitionen (vgl. Tabelle 3).

Abgesehen von „Ausreißern“ nach oben wie nach unten können aus den Ergebnissen von Tabelle 3 einige allgemeine Aussagen abgeleitet werden. Mit Ausnahme der aktuellen ungarischen Minderheitsregierung überstanden alle Koalitionen in Drei- und Vierparteiensystemen ihre gesamte Wahlperiode. Allerdings muss das neue polnische Kabinett von Premierminister Donald Tusk seine Überlebensfähigkeit erst noch beweisen. Bislang deuten viele Signale auf eine deutlich größere Bereitschaft zur Kompromissfindung hin, als dies den Vorgängerregierungen zu attestieren war. Umgekehrt zerbrachen alle drei Regierungsbündnisse, die unter den Verhältnissen von sieben und mehr Parteien zustande kamen. Die slowakische Regierungskoalition (1994 bis 1998) konnte sich allerdings nach ihrer zwischenzeitlichen Auflösung neu konstituieren. Bei jenen Wahlen, die für fünf Parteien zum Parlamentseinzug reichten, hielten zwei von vier Koalitionen bis zum Ablauf der Amtszeiten. In Polen wurde das konservativ-liberale Regierungsbündnis aus dem AWS und der Freiheitsunion (UW) im Jahr 2000 vorzeitig beendet. Die erste Regierung der Slowakei (1992 bis 1994) zerbrach nach reichlich zwei Jahren. Für die amtierende tschechische Regierung kann bisher nur als Halbzeitbilanz Stabilität festgestellt werden. Für Sechsparteiensysteme fällt die Beurteilung ebenfalls gemischt aus: Von acht Wahlperioden ${ }^{31}$ hielten die

29 Vgl. Giovanni Sartori, Parties and Party Systems: A Framework for Analysis, Cambridge 1976, S. 316.

30 Vgl. Thomas Saalfeld, Parteiensystem und Kabinettsstabilität in Westeuropa 1945 - 1999, in: Oskar Niedermayer / Richard Stöss / Melanie Haas (Hrsg.), a.a.O. (Fn. 17), S. 477 - 506, S. 489.

31 Für die aktuelle Regierung in der Slowakei kann dahingehend noch keine Aussage getroffen werden, doch scheint die Zukunft des fragilen Bündnisses aus der linkspopulistischen Partei Smer (Richtung) von Premierminister Robert Fico, der HZDS und der SNS aufgrund ihrer geringen programmatischen Kompatibilität ungewiss. 


\begin{tabular}{|c|c|c|}
\hline Parteienanzahl* & Wahlperiode & Koalitionsstabilität \\
\hline 3 & Ungarn (2002-2006) & kein Koalitionsbruch \\
\hline 4 & Tschechien $(1990-1992)^{* *}$ & kein Koalitionsbruch \\
\hline 4 & Tschechien (2002-2006) & kein Koalitionsbruch \\
\hline 4 & Ungarn (seit 2006) & Koalitionsbruch \\
\hline 4 & Polen (seit 2007) & bisher kein Koalitionsbruch \\
\hline 5 & Slowakei $(1990-1992)^{* *}$ & kein Koalitionsbruch \\
\hline 5 & Slowakei (1992-1994) & Koalitionsbruch und vorgezogene Neuwahlen \\
\hline 5 & Polen (1997-2001) & Koalitionsbruch \\
\hline 5 & Tschechien (1998-2002) & kein Koalitionsbruch \\
\hline 5 & Tschechien (seit 2006) & bisher kein Koalitionsbruch \\
\hline 6 & Ungarn (1990-1994) & kein Koalitionsbruch \\
\hline 6 & Tschechien (1992-1996) & kein Koalitionsbruch \\
\hline 6 & Polen (1993-1997) & Koalitionsbruch \\
\hline 6 & Ungarn (1994-1998) & kein Koalitionsbruch \\
\hline 6 & Tschechien (1996-1998) & Koalitionsbruch und vorgezogene Neuwahlen \\
\hline 6 & Slowakei (1998-2002) & kein Koalitionsbruch \\
\hline 6 & Ungarn (1998-2002) & kein Koalitionsbruch \\
\hline 6 & Polen (2005-2007) & Koalitionsbruch und vorgezogene Neuwahlen \\
\hline 6 & Slowakei (seit 2006) & bisher kein Koalitionsbruch \\
\hline 7 & Slowakei (1994-1998) & zwischenzeitlicher Koalitionsbruch \\
\hline 7 & Slowakei (2002-2006) & Koalitionsbruch und vorgezogene Neuwahlen \\
\hline 29 & Polen (1991-1993) & Koalitionsbruch und vorgezogene Neuwahlen \\
\hline \multicolumn{3}{|c|}{$\begin{array}{l}\text { * Die Werte der Parteienanzahl entsprechen den Ergebnissen der Parlamentswahlen. Zahlenmäßige Ver } \\
\text { änderungen während einer Legislaturperiode bleiben unberücksichtigt; sie verändern die Gesamtergeb } \\
\text { nisse nur minimal. } \\
\text { ** Angaben jeweils für den tschechischen und den slowakischen Teil der Tschechoslowakei. } \\
\text { Quelle: Eigene Zusammenstellung aus verschiedenen Quellen (siehe Tabelle 1). }\end{array}$} \\
\hline
\end{tabular}

Regierungen in fünf Fällen bis zu ihrem regulären Ende. Für alle Regierungskoalitionen, die ohne Auflösungen oder Umbildungen ihre Legislaturperioden absolvierten, liegt der Medianwert an Parlamentsparteien bei 5,18. Zerbrach hingegen das Regierungsbündnis vorzeitig, waren laut Medianwert sechs Fraktionen parlamentarisch beteiligt. Gleichwohl sind die Unterschiede der Kabinettsstabilität zwischen Tschechien und Ungarn einerseits sowie Polen und der Slowakei andererseits nicht minder auffällig und reduzieren somit die allgemeine Aussagefähigkeit der reinen Parteienanzahl.

Die Folgen der Parteienkonzentration sind jedoch nicht nur hinsichtlich des Stabilitätsgedankens interessant, sondern ebenso in Bezug auf die Frage nach effektiver Regierungsbildung und funktionierender Kabinettsarbeit. Dafür ist es notwendig, die unterschiedliche programmatische Kompatibilität der Parlaments- beziehungsweise Regierungsparteien zu 
beleuchten. Hierbei spricht einiges für die Hypothese, dass eine größere Parteienkonzentration zu weniger ideologischer und inhaltlicher Heterogenität führt. Vor allem Anti-SystemParteien, die als potenzielle Koalitionspartner der demokratischen Kräfte ausfallen, erzwangen in der Vergangenheit die Bildung von parlamentarischen Mehrheiten, die sachpolitisch und kulturell weit auseinander lagen. ${ }^{32}$ Besonders das Ausscheiden der extremistischen Parteien in Polen (Samoobrona und LPR), Tschechien (Republikaner), Ungarn (MIÉP) und zumindest vorübergehend in der Slowakei (SNS, 2002 bis 2006) erhöhte damit deutlich den Grad an programmatischer Homogenität. In der Slowakei wäre eine Fortsetzung der demokratischen Vier-Parteien-Koalition nach der Wahl 2002 ohne das Ausscheiden der zeitweise gespaltenen nationalistischen SNS nicht möglich gewesen.

Nicht weniger problematisch verhielt es sich, wo extremistische Parteien in die Regierungsverantwortung gelangten. Die erste unabhängige Regierung der Slowakei scheiterte, nachdem ein Flügel der nationalistischen SNS die Partei Ende 1993 verließ und Ministerpräsident Vladimir Mečiar seine Mehrheit verlor. Auch die Neuauflage (1994 bis 1998) des fragilen Bündnisses unter Einbeziehung der Vereinigten Slowakischen Arbeiterpartei (ZRS) wurde von dauerhaften Koalitionskrisen begleitet. Die Allianz in Polen (2005 bis 2007) stand angesichts der Regierungsunfähigkeit gleich zweier antidemokratischer Juniorpartner von Anfang an unter keinem guten Stern. In politischen Sachfragen blockierten sich die Koalitionäre dauerhaft und brachten das Bündnis mehrfach vor seinem vorzeitigen Ende an den Rand des Scheiterns. Im September 2006 zerbrach die Koalition im Streit um den polnischen Haushalt 2007 und die angekündigte Entsendung von 1000 Soldaten nach Afghanistan. Die Mehrheit der Samoobrona-Fraktion verließ die Regierung, nachdem sie mit ihren Forderungen, auf das Engagement am Hindukusch zu verzichten und im kommenden Jahr mehr Geld für die Landwirtschaft auszugeben, gescheitert war. Da es der PiS allerdings nicht gelang, eine Mehrheit mit anderen Partnern auf die Beine zu stellen und Meinungsumfragen herbe Verluste für alle drei Regierungsparteien im Falle von Neuwahlen voraussagten, kehrte Samoobrona nach nur einem Monat so überraschend in die Regierung zurück, wie sie das Kabinett verlassen hatte. ${ }^{33}$

Doch nicht nur programmatische Kompatibilität der verbliebenen Akteure in den Parlamenten steigt mit der Parteienkonzentration, sondern auch die Zahl gegenläufiger Konfliktlinien nimmt ab. Mit dem Ausscheiden der extremistischen Parteien hat vor allem das Cleavage der Pro- und Anti-System-Parteien an Bedeutung verloren. So führten die verschiedenen Konfliktlinien im rechten politischen Spektrum Polens in den 1990er Jahren zu einer Zersplitterung des Post-Solidarność-Lagers. Im AWS versammelten sich Wirtschaftsliberale, konservative, klerikal-nationale und auch rechtsextreme Kräfte, deren divergierende Richtungen eine Output-orientierte Regierungspolitik konterkarierten. Im Sejm stimmten die einzelnen Abgeordneten meist abweichend und nach den Präferenzen ihrer Untergruppierungen, was den Erosionsprozess der Regierung unter Jerzy Buzek beschleunigte. In der Slowakei saßen gar die sozialistische ZRS und die antikommunistische SNS in einer gemeinsamen Regierung (1994 bis 1998). Zwar bleiben nach dem Ausscheiden sol-

32 Die Tolerierung der bürgerlich-konservativen Koalition unter Ministerpräsident Vaćlav Klaus (1996 bis 1998) durch die ČSSD ist ebenso zu erwähnen wie die demokratische Regenbogenkoalition unter Premier Mikulás Dzurinda.

33 Vgl. Thomas Urban, Kaczynskis Dacapo. Polens Premierminister lässt mangels Alternative die kaputte Koalition wiederauferstehen, in: Süddeutsche Zeitung vom 18. Oktober 2006, S. 8. 
cher „Klientel“-Parteien deren Interessen durch die inhaltliche Integrierung durch andere Parteien erhalten, doch ist dies in aller Regel mit einer deutlichen Abmilderung des Konfliktpotentials verbunden. Die Verkleinerung der Parteiensysteme fördert generell eine Harmonisierung des politischen Wettbewerbs und erhöht dadurch die Kooperations- und Koalitionsfähigkeit der politischen Akteure. ${ }^{34}$ Allerdings sind weitere Faktoren, beispielsweise eine steigende Bündnisfähigkeit durch neue, gewandelte beziehungsweise unvorbelastete Akteure, zu berücksichtigen. Die für westeuropäische Parteiensysteme aufgestellte Kurzformel „mehr Pluralität - weniger Polarisierung“35 gilt (mit Ausnahme Ungarns) in den ostmitteleuropäischen Staaten nicht.

Dennoch sind die grundsätzlichen Annahmen steigender Regierungseffektivität bei abnehmender Parteienanzahl im konkreten Einzelfall Einschränkungen unterworfen, die allgemeine Aussagen verbieten. Mit der Parteienkonzentration in Ungarn stieg die Polarisierung der zwei rivalisierenden Lager, so dass gesamtgesellschaftliche Entscheidungen blockiert werden. So konnten sich die beiden zerstrittenen Blöcke seit Ende des Kommunismus nicht auf eine ausstehende Totalrevision der Verfassung einigen, da eine verfassungsändernde Zwei-Drittel-Mehrheit des Parlaments stets verfehlt wurde. Ausgerechnet das bislang als konzentriert und stabilisiert charakterisierte tschechische Parteiensystem ist hinsichtlich einer effektiven Regierungsarbeit stark beschränkt. Die als Anti-System-Partei geltende KSČM erzielte bei allen bisherigen Wahlen ein zweistelliges Ergebnis, wodurch klare und koalitionsfähige Mehrheiten des rechten oder linken Lagers meist behindert wurden. Da sich die beiden Großparteien ODS und ČSSD nicht auf eine formale Zusammenarbeit einigen konnten, mussten seit 1996 dreimal Minderheitsregierungen gebildet werden. ${ }^{36}$ Erfolgreiche Regierungsarbeit im Sinne der politischen Output-Dimension ist unter derart labilen Mehrheitsverhältnissen nur bedingt möglich, so dass die Umsetzung notwendiger wirtschafts- und sozialpolitischer Reformen in Tschechien seit langem blockiert ist.

\section{Stabilität durch Wandel oder Kontinuität der Instabilität?}

Die Parteiensysteme in Ostmitteleuropa haben in den vergangenen knapp zwei Jahrzehnten vielfältige Wandlungsprozesse durchlaufen. Dies ist einerseits den tiefgreifenden politischen, ökonomischen und kulturellen Veränderungen im Transformationsverlauf geschuldet. Andererseits wandelten sich die Parteiensysteme aufgrund der verkürzten Demokratisierungsphase im Vergleich zu Westeuropa deutlich schneller und dynamischer. Als eine der zentralen Veränderungen ist die konstant zunehmende Parteienkonzentration zu nennen, gemessen an der Zahl der Parteien und den Stimmenanteilen, die die größten auf sich vereinigen. Waren in den 1990er Jahren meist sechs und mehr Parteien in den nationalen Parlamenten vertreten, sind momentan durchschnittlich weniger als fünf Fraktionen in den Volksvertretungen zu finden. Lag der addierte Stimmenanteil der jeweils vier stärksten Parteien vor zehn Jahren bei 77,3 Prozent, so gewinnen sie heute zusammen im Schnitt mehr

34 Vgl. Herbert Kischelt / Zdenka Mansfeldová / Radoslaw Markowski / Gábor Tóka, Post-Communist Party Systems. Competition, Representation, and Inter-Party Cooperation, Cambridge 1999, S. $303-306$.

35 Oskar Niedermayer / Richard Stöss / Melanie Haas, a.a.O. (Fn. 17), S. 32.

36 Vgl. Florian Grotz, Stabile Regierungsbündnisse? Determinanten der Koalitionspolitik in Ostmitteleuropa, in: Osteuropa, 57. Jg. (2007), H. 4, S. 109 - 122, S. 115 f. 
als 88 Prozent an Zustimmung. Die Slowakei stellt hierbei eine doppelte Ausnahme dar, da nach den antidemokratischen Abwegen der 1990er Jahre die Parteiensystemkonsolidierung verspätet einsetzte und bislang keine Konzentrationseffekte zu beobachten sind.

Dies blieb nicht wirkungslos auf die Entwicklung der Parteiensysteme in den vier Ländern. Das polnische durchlebte seit 1991 einen konstanten Konzentrationsprozess vom ausgeglichenen Mehrparteiensystem (1991 bis 1993) über ein Mehrparteiensystem mit zwei dominanten Parteien (1993 bis 2001), ein Mehrparteiensystem mit einer dominanten Partei (2001 bis 2005) bis zum momentanen Mehrparteiensystem mit zwei dominanten Parteien. In Tschechien und Ungarn entstanden bereits Ende der 1990er Jahre Mehrparteiensysteme mit zwei dominanten Parteien. Nur in der Slowakei änderte sich an der Einordnung in die Parteiensystemtypologie als ausgeglichenes Mehrparteiensystem seit Beginn der staatlichen Unabhängigkeit nichts.

Doch führte die konzentrationsbedingte Wandlung der Parteiensysteme in Ostmitteleuropa zu stabileren Verhältnissen und effektiverer Regierungsarbeit? In Polen funktionierte bisher unter den Bedingungen keines Parteiensystemtyps krisenfreie Regierungspolitik, wobei sich die Vorzeichen dafür seit den Wahlen 2007 verbessert haben. Die Regierungsbildung in Tschechien wird zwar nach wie vor durch die Mandate der KSČM behindert, doch die Stabilität des Parteiensystems nahm mit der Entstehung zweier rivalisierender Großparteien zu. Auch in Ungarn bestimmen zwei rivalisierende Machtblöcke das Parteiensystem. Die Regierungsstabilität war allerdings hier schon vor dem Konzentrationsschub 2002 gegeben. Der gleichbleibende Parteiensystemtyp in der Slowakei führte nicht zu einer Abnahme der instabilen Regierungsverhältnisse.

In den Parteiensystemen Ostmitteleuropas sind Stabilität und Wandel zwar keine Gegensätze, dennoch dürfen die Auswirkungen einer steigenden Parteienkonzentration nicht überschätzt werden und zur Annahme des Umkehrschlusses „Stabilität durch Wandel“ führen. Die Entwicklung von stark fluktuierenden Parteiensystemen zu gefestigten und konzentrierten Wettbewerbskonstellationen begünstigte vor allem in der Frühphase des Transformationsprozesses eine Zunahme an parlamentarischer Stabilität und Output-orientierter Effektivität. Die Leistungsbilanz der Regierungen hängt allerdings von deutlich mehr Faktoren als der bloßen Anzahl an Parlamentsparteien ab. In diesem Zusammenhang muss auf das individuelle (Fehl-)Verhalten der politischen Eliten ebenso wie auf die jeweiligen innergesellschaftlichen Konfliktstrukturen hingewiesen werden. Daher lässt sich auch nicht auf ein generelles Ende der Veränderungsprozesse in den vier Parteiensystemen schließen. Parteiallianzen, Umformierungen, Fraktionsaus- und -übertritte oder die Etablierung neuer parteipolitischer Akteure könnten weiterhin eine wichtige Rolle bei der Entwicklung der Parteiensysteme in Polen, Tschechien, der Slowakei und Ungarn spielen. Dies wird maßgeblich von der Problemlösungskompetenz des jeweiligen politischen Personals abhängen. Einen Blankoscheck für dauerhafte parlamentarische Beteiligung bedeutet die Parteienkonzentration nicht; sie ist ebenso wenig als Votum für die übrig gebliebenen Akteure zu bewerten. Die zunehmende Parteienkonzentration in Ostmitteleuropa ist nicht nur die Ursache einer wachsenden parlamentarischen Stabilität, sondern ebenso Folge der bisherigen Instabilität. Deren Auswirkungen sind sich die Wähler in den vier Staaten zunehmend bewusst geworden. Den momentanen Vertrauensvorschuss könnten sie allerdings bei Enttäuschung ihrer (vor allem ökonomischen) Erwartungen mit neuerlichen Proteststimmen an zwielichtige Populisten bestrafen. 